\title{
Ebola in West Africa - ICAN meeting November 2014 - ICPIC Outstanding seminar series
}

Didier Pittet ${ }^{1 *}$ and Andreas Voss ${ }^{2}$

\begin{abstract}
During the 2014 Infection Control Africa Network (ICAN) meeting in Harare, videos were recorded for this seminar series on Ebola in West Africa. This summary is an introduction into the topic and includes the links to the produced video clips.
\end{abstract}

Keywords: Ebola, ICAN, Video link

The Infection Control Africa Network (ICAN) conference is the most prominent annual infection prevention and control (IPC) meeting in Africa. ICAN is the largest IPC organization in Africa and is committed to support countries and organizations involved in establishing infection prevention and control activities throughout the African continent (www.icanetwork.co.za/).

In 2014, the 5th ICAN conference was held from 35th November 2014 in Harare, Zimbabwe.

The main topic for discussion and sharing of expertise during the 5th ICAN conference was Ebola. Several aspects of the management of the ongoing Ebola epidemic in West Africa were discussed. Lectures by key field experts and interviews conducted by Professor Didier Pittet in Harare were video-recorded and are now accessible from the HUG-WHO Collaborating Centre on Patient Safety in Geneva, Switzerland (http://tinyurl. com/EbolaWestAfricaICPICsem2014)

This high-level material is part of the International Conference on Prevention \& Infection Control (ICPIC) Outstanding Seminar Series (www.icpic.com/ or www. icpic2015.com).

Exclusive interviews by Prof. Didier Pittet of:

Prof. Shaheen Mehtar, Chair of the Infection Control Africa Network (ICAN), encountered cases of hemorrhagic fever for the first time in 1980. She shares her long-lasting experience, the recent difficulties facing the current

\footnotetext{
* Correspondence: Didier.Pittet@hcuge.ch

${ }^{1}$ Infection Control Programme and WHO Collaborating Centre on Patient Safety, University of Geneva Hospitals and Faculty of Medicine, 4 Rue Gabrielle-Perret-Gentil, 1211, Geneva 14, Switzerland

Full list of author information is available at the end of the article
}

epidemic in West Africa, and the future of Ebola control in Africa (http://tinyurl.com/EbolaAfritwMethar).

Prof. Babacar Ndoye, expert in IPC and microbiology, and consultant for the WHO, undertook a mission to evaluate health-care systems in Guinea and Liberia, where he visited most hospitals and provided bedside expertise. He reports on the realities from the field, compares health systems weaknesses and improvements, and provides perspectives on infection and Ebola control in Africa (http://tinyurl.com/EbolaAfritwNdoye1) (http:// tinyurl.com/EbolaAfritwNdoye2).

Prof. Folasade Ogunsola, professor of medical microbiology at the College of Medicine, University of Lagos, who was involved with the Ebola outbreak response in Nigeria (http://tinyurl.com/EbolaAfritwSade).

Dr. Frederick Marais, from the Western Cape government in South Africa, part time lecturer at Stellenbosch University, has developed a "community protocol" to engage the community and community leaders. The approach includes culture values, customs and practices, and identifies steps undertaken from the perspective of disease control that might be culturally insensitive (http:// tinyurl.com/EbolaAfritwMarais).

Keynote lectures include:

Ebola Outbreak: Lessons learned

Professor Shaheen Mehtar, Chair of the Infection Control Africa Network (ICAN)

(http://tinyurl.com/EbolaAfrMethar)

Ebola outbreak and infection prevention and control: Experience from Guinea

Professor Babacar Ndoye, Consultant for WHO, expert in IPC and Microbiology

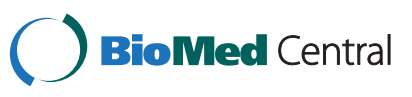


(http://tinyurl.com/EbolaAfrNdoye)

Community-engaged IPC: Essential steps towards disease containment

Dr. Frederick Marais, Western Cape Government, Lecturer at Stellenbosch University

(http://tinyurl.com/EbolaAfrMarais)

Faculties, lectures and learning objectives

The learning objectives are:

- To understand the major aspects associated with the control of the Ebola virus disease outbreak in West Africa between April 2014 and December 2014

- To review the major challenges for healthcare systems and in particular in regards to the application of basic and adapted infection control measures

- To learn about the local population's possible resistance toward field actions engaged by governments, MSF, WHO and NGOs to control the Ebola outbreak

- To understand differences between the Liberian, Guinean, Sierra Leonean and Nigerian responses to the Ebola outbreak

- To identify steps in the perspective of Ebola virus disease control that might be culturally insensitive and better engage with the community and community leaders to control Ebola

Professor Shaheen Mehtar, Chair of the Infection Control Africa Network (ICAN), shares her long-lasting experience, the recent difficulties and the future of Ebola control in Africa. The recent Ebola epidemic has been different from the other because there has been a huge "explosion" coming from a silent epidemic among the Great Apes. In the current situation, Ebola has reached a very dense population and large cities in West Africa. Ebola also came to the international attention once highincome countries healthcare workers got infected with the disease. Prof. Mehtar shares 3 important lessons learned from the current epidemic: i) Involve the community ii) Improve surveillance systems iii) Use PPE provided by WHO. Training and education are the most important parameters for Ebola control for both the HCWs and the community. "It is now the perfect time to mobilize international communities to contribute in R\&D including vaccines by funding it through international public money." (http://tinyurl.com/EbolaAfrMethar)

Professor Babacar Ndoye, consultant for WHO, physician and biologist from Senegal, expert in IPC and microbiology, shares his experience in Guinea during the current Ebola outbreak. Prof. Ndoye went to Guinea to assess the level of preparedness facilities to handle the situation. At first, he encountered difficulties to speak about IPC and most of the people could not understand his role. However, after several months, Prof. Ndoye was able to monitor progress and see the impact of his visits. Hospital staff were finally aware of the importance of IPC in all facilities. However, the general population's resistance to IPC was very strong in certain parts of the country. Prof. Ndoye observed that IPC is still very weak in general, in particular in regards to Ebola control. Facilities have to be suitable to effectively face up to the epidemic, and to be able to prevent HAI. His main recommendations would be to create local conditions in facilities to implement sustainable professional practices in accordance with the technical recommendations provided. A roadmap is proposed to achieve this. Of course, resources are necessary to ensure that these recommendations are implemented. (http://tinyurl.com/EbolaAfrNdoye)

Dr. Frederick Marais, a member Infection Control Africa Network (ICAN), shares his expertise on the need for community involvement in the fight against diseases and specifically against Ebola. Dr. Marais has been studying the community component since 2000 and found limitations in conventional biomedical approaches. Through the CARE model, he hopes to provide a solution to generate better responses against infectious diseases such as Ebola. A key missing link in the Ebola control and response is community engagement. Collaboration with identified key community leaders must be implemented for proper IPC and community partnership. With the "community protocol" including culture values, customs and practices defined by Dr. Marais, it is possible to identify steps that are rational from the perspective of disease control, but which might be culturally insensitive. "Through debate and dialogue, by building trust with reciprocal learning and community entry with cultural humility, we will come with a midway solution. "For Dr. Marais, "it is important to work with the community, think beyond Ebola, and build a structure and knowledge."

(http://tinyurl.com/EbolaAfritwMarais).

Competing interests

The authors declare that they have no competing interests.

\section{Authors' contributions}

DP drafted the manuscript and lead the interviews during the filming. AV supported the filming and reviewing of the manuscript. Both authors read and approved the final manuscript.

\section{Author details}

${ }^{1}$ Infection Control Programme and WHO Collaborating Centre on Patient Safety, University of Geneva Hospitals and Faculty of Medicine, 4 Rue Gabrielle-Perret-Gentil, 1211, Geneva 14, Switzerland. ${ }^{2}$ Infection Control, Radboud University Medical Centre and Canisius-Wilhelmina Hospital, Nijmegen, The Netherlands.

Received: 9 April 2015 Accepted: 9 April 2015

Published online: 12 May 2015 